\title{
Identification and Characterization of 40 Isolated Rehmannia glutinosa MYB Family Genes and Their Expression Profiles in Response to Shading and Continuous Cropping
}

\author{
Fengqing Wang ${ }^{1, \dagger}$, Yanfei Suo ${ }^{1, \dagger}$, He Wei ${ }^{2, \dagger}$, Mingjie $\mathrm{Li}^{3}$, Caixia Xie ${ }^{4}$, Lina Wang ${ }^{4}$, \\ Xinjian Chen ${ }^{1}$ and Zhongyi Zhang ${ }^{1,3, *}$
}

1 College of Agronomy, Henan Agricultural University, Zhengzhou 450002, China; E-Mails: heauzycxw@126.com(F.W.); suoyanfei1990@163.com(Y.S.); chenxinjian3978@126.com (X.C.)

2 Institute of Industrial Crops, Henan Academy of Agricultural Science, Zhengzhou 450002, China; E-Mail: weihezj123@126.com

3 College of Crop Sciences, Fujian Agriculture and Forestry University, Fuzhou 350002, China; E-Mail: xinyuzszj@163.com

4 School of medicine, Henan University of Traditional Chinese Medicine, Zhengzhou 450046, China; E-Mails: nanyangxcx@126.com (C.X.); lnsdhz2012@126.com (L.W.)

$\dagger$ These authors contributed equally to this work.

* Author to whom correspondence should be addressed; E-Mail: zyzhang@fafu.edu.cn; Tel./Fax: +86-591-8374-2793.

Academic Editor: Lam-Son Phan Tran

Received: 12 May 2015 / Accepted: 23 June 2015 / Published: 2 July 2015

\begin{abstract}
The v-myb avian myeloblastosis viral oncogene homolog (MYB) superfamily constitutes one of the most abundant groups of transcription factors (TFs) described in plants. To date, little is known about the MYB genes in Rehmannia glutinosa. Forty unique MYB genes with full-length cDNA sequences were isolated. These 40 genes were grouped into five categories, one R1R2R3-MYB, four TRFL MYBs, four SMH MYBs, 25 R2R3-MYBs, and six MYB-related members. The MYB DNA-binding domain (DBD) sequence composition was conserved among proteins of the same subgroup. As expected, most of the closely related members in the phylogenetic tree exhibited common motifs. Additionally, the gene structure and motifs of the $R$. glutinosa MYB genes were analyzed. MYB gene expression was analyzed in the leaf and the tuberous root under two abiotic
\end{abstract}


stress conditions. Expression profiles showed that most $R$. glutinosa MYB genes were expressed in the leaf and the tuberous root, suggesting that MYB genes are involved in various physiological and developmental processes in $R$. glutinosa. Seven MYB genes were up-regulated in response to shading in at least one tissue. Two MYB genes showed increased expression and 13 MYB genes showed decreased expression in the tuberous root under continuous cropping. This investigation is the first comprehensive study of the MYB gene family in R. glutinosa.

Keywords: MYB transcription factor; shading; continuous cropping; Rehmannia glutinosa

\section{Introduction}

MYB transcription factors (TFs), a group of TFs with conserved DNA binding domains, are widely distributed in all eukaryotic organisms and constitute one of the largest TF families in the plant kingdom. MYB TFs contain a conserved DNA-binding domain (DBD) that is homologous to the DBD of animal c-Myb [1]. This domain generally consists of 1-4 imperfect amino acid sequence repeats (R0, R1, R2, and R3) of approximately 52 amino acids, each encoding three $\alpha$-helices [2]. The second and third helices form a helix-turn-helix $(\mathrm{HTH})$ structure [3] that intercalates in the major groove when bound to DNA. The third $\alpha$-helix is thought to play a recognition role in binding to a short DNA sequence [4]. MYB proteins can be classified into 4R-MYB (contain four R1/R2-like repeats), R1R2R3-type MYB (3R-MYB), R2R3-MYB and MYB-related (contain single or a partial MYB repeat) depending on the number of adjacent repeats in the MYB domain (one, two, three or four) [2].

The first identified gene encoding a MYB domain protein in plants was COLORED1 $(\mathrm{Cl})$, which is involved in anthocyanin biosynthesis in the aleurone layer of Zea mays [5]. An increasing number of plant MYB TF members have been identified and characterized in numerous plant species. Most plant MYB genes encode R2R3-MYB class proteins, which contain two repeats $[2,6]$. These proteins are thought to have evolved from an R1R2R3-MYB ancestor by the loss of the sequences encoding the $\mathrm{R} 1$ repeat and subsequent expansion of the family [7,8]. The diverse functions of these genes in plant-specific processes include primary metabolism [9-12], secondary metabolism [13-16], cell fate and identity [17-21], environmental stresses [22-25], and organ development [26-32].

Rehmannia glutinosa, one of the most famous Four Huai medicines, has been widely used as a medicinal herb with a long history of cultivation and application in China. The R. glutinosa genome has not yet been sequenced. There are only limited reports on the characterization of protein coding genes and non-coding miRNAs in $R$. glutinosa [33-36]. In contrast with the intensive research on MYB TFs in both model and crop plants such as Arabidopsis thaliana, Oryza sativa, Glycine max and Z. mays [2,37-39], there is no reported characterization of the MYB TFs in R. glutinosa. In this study, we identified a comprehensive set of MYB family members in $R$. glutinosa. Phylogenies, secondary protein structures, and gene expression patterns were analyzed and compared between conventional and novel classes of MYB candidates. These results will provide valuable information for understanding the classification and putative functions of $R$. glutinosa MYBs and other TF families 
and increase our understanding of potential transcriptional regulatory mechanisms in the development of the $R$. glutinosa tuberous root.

\section{Results and Discussion}

\subsection{Identification of the MYB Gene Family in R. glutinosa}

To predict $R$. glutinosa MYB genes at the transcriptome level, we downloaded all 197 Arabidopsis MYB cDNA sequences from GenBank (http://www.ncbi.nlm.nih.gov/genbank). BLASTn analysis of Arabidopsis MYBs against the current transcriptome database of $R$. glutinosa [35] was then performed using the BLASTn algorithm [40]. An $e$-value cut-off of $1 \times 10^{-5}$ was applied. As a result, a total of 165 MYB unigenes of $R$. glutinosa were identified. Among the 165 unigenes, the lengths of 56 (about one third) sequences were between 200 and $500 \mathrm{bp}$. The other 109 sequences were longer than $500 \mathrm{bp}$. Forty sequences possessed complete opening reading frames (ORF) in ORF Finder (Tables 1 and $\mathrm{S} 1$ ). The remaining $R$. glutinosa MYB genes possessing incomplete ORFs were excluded from further analysis.

To validate the $M Y B$ candidates obtained using BLASTn, the proteins encoded by the 40 matching unigenes were analyzed using the online Batch CD-search tool (CDD, in NCBI) [41] and the SMART database [42]. Based on these results, all 40 MYB genes were confirmed to be MYB candidates by at least one of these two tools.

We also searched the NCBI database for plant kingdoms with identified MYB protein amino acid sequences and found that thirty-six sequences showed high identity (52\%-91\%) with Sesamum indicum, which belongs to the family Pedaliaceae. The other three genes, $\operatorname{Rg} M Y B 2, \operatorname{Rg} M Y B 3$ and $\operatorname{Rg} M Y B 18$, matched to Salvia miltiorrhiza, which belong to the family Labiatae (Table 1). Another gene, RgMYB6, showed the highest identity with Scutellaria baicalensis (Labiatae). The three families Pedaliaceae, Labiatae and Scrophulariaceae belong to Tubiflorae Sympetala.

\subsection{Protein Motif and Structure Analysis of the MYBs in R. glutinosa}

To further reveal the divergence of the $R$. glutinosa MYB proteins, putative motifs were predicted by the program multiple em for motif elicitation (MEME), and 10 distinct motifs were identified. The positions of the MYB/SANT domains and any conserved motifs are shown in Figure 1. Motif 2 was found in all 40 MYB proteins. Simple Modular Architecture Research Tool (SMART) analysis revealed that seven motifs (motif 1, 2, 3, 4, 5, 9 and 10) are conserved MYB DNA-binding domains (DBD), motif 6 is a coiled coil region, motif 7 is a H15 domain, and motif 8 is an unknown function domain. 
Table 1. MYB genes identified in Rehmannia glutinosa transcriptome.

\begin{tabular}{|c|c|c|c|c|}
\hline Gene Name & Gene ID & $\begin{array}{l}\text { Gene } \\
\text { Length/bp }\end{array}$ & $\begin{array}{l}\text { Amino } \\
\text { Acid } \\
\text { Length }\end{array}$ & Blast Results (Query Cover, $e$-Value, Identities, Accession No., Description, (Species)) \\
\hline $\operatorname{RgMYB1}$ & CL429Contig1 & 1126 & 273 & $95 \%, 2 \times 10^{-144}, 76 \%, \mathrm{XP} \_011070362.1$, transcription repressor MYB5-like (Sesamum indicum) \\
\hline $\operatorname{Rg} M Y B 2$ & CL8750Contig1 & 831 & 210 & $90 \%, 2 \times 10^{-95}, 73 \%$, AGN52078.1, MYB-related transcription factor (Salvia miltiorrhiza) \\
\hline $\operatorname{RgMYB3}$ & Unigene 7708 & 1036 & 318 & $100 \%, 0.0,79 \%$, AGN52106.1, MYB-related transcription factor (S. miltiorrhiza) \\
\hline $\operatorname{Rg} M Y B 4$ & CL128Contig2 & 1582 & 418 & $100 \%, 0.0,70 \%$, XP_011087570.1, transcription factor MYB86-like (S. indicum) \\
\hline RgMYB5 & Unigene19474 & 673 & 199 & $95 \%, 2 \times 10^{-89}, 72 \%$, XP_011079754.1, transcription factor MYB114-like (S. indicum) \\
\hline RgMYB6 & Unigene 18939 & 897 & 267 & $97 \%, 1 \times 10^{-96}, 59 \%$, AGZ16400.1 MYB7 (Scutellaria baicalensis) \\
\hline $\operatorname{Rg} M Y B 7$ & CL7757Contig 1 & 1207 & 306 & $100 \%, 8 \times 10^{-118}, 61 \%$, XP_011086359.1, MYB-related protein Zm1 (S. indicum) \\
\hline $\operatorname{Rg} M Y B 8$ & Unigene14277 & 1100 & 299 & $100 \%, 4 \times 10^{-161}, 73 \%, \mathrm{XP}_{-} 011098622.1, \mathrm{MYB}-\mathrm{related}$ protein $306-$ like $(S$. indicum $)$ \\
\hline $\operatorname{Rg} M Y B 9$ & Unigene 7159 & 1338 & 319 & $100 \%, 1 \times 10^{-153}, 74 \%, \mathrm{XP}_{-} 011071639.1, \mathrm{MYB}-$ related protein $306-$ like $(S$. indicum $)$ \\
\hline $\operatorname{RgMYB10}$ & Unigene 16684 & 1017 & 247 & $100 \%, 4 \times 10^{-92}, 63 \%, \mathrm{XP} \_11080041.1$, MYB-related protein Myb4-like (S. indicum) \\
\hline $\operatorname{RgMYB11}$ & Unigene9736 & 1347 & 251 & $100 \%, 4 \times 10^{-149}, 83 \%, \mathrm{XP}_{-} 011091315.1$, MYB-related protein Myb4-like (S. indicum) \\
\hline $\operatorname{RgMYB12}$ & Unigene 12270 & 862 & 265 & $99 \%, 3 \times 10^{-71}, 52 \%$, XP_011091328.1, MYB-related protein Myb4-like $(S$. indicum $)$ \\
\hline $\operatorname{RgMYB13}$ & Unigene12354 & 1307 & 292 & $97 \%, 5 \times 10^{-95}, 56 \%, \mathrm{XP}_{-} 011100326.1$, MYB-related protein Myb4-like (S. indicum) \\
\hline $\operatorname{RgMYB14}$ & Unigene21429 & 1227 & 293 & $98 \%, 1 \times 10^{-105}, 59 \%, X_{-}^{-} 011100326.1$, MYB-related protein Myb4-like (S. indicum) \\
\hline $\operatorname{Rg} M Y B 15$ & CL5174Contig3 & 1101 & 219 & $94 \%, 2 \times 10^{-84}, 68 \%, \mathrm{XP} \_011089822.1$, transcription factor MYB48-like (S. indicum) \\
\hline $\operatorname{RgMYB16}$ & CL9333Contig1 & 1327 & 286 & $100 \%, 4 \times 10^{-139}, 75 \%, \mathrm{XP}_{-} 011076599.1$, transcription factor MYB108-like $(S$. indicum $)$ \\
\hline $\operatorname{Rg} M Y B 17$ & Unigene 5899 & 1217 & 288 & $96 \%, 1 \times 10^{-131}, 74 \%$, XP_011070788.1, transcription factor MYB24 (S. indicum) \\
\hline $\operatorname{Rg} M Y B 18$ & CL6949Contig1 & 790 & 202 & $91 \%, 8 \times 10^{-94}, 74 \%$, AGN52041.1, MYB-related transcription factor (S. miltiorrhiza) \\
\hline $\operatorname{Rg} M Y B 26$ & CL3700Contig2 & 3518 & 1015 & 100\%, 0.0, 75\%, XP_011081822.1, MYB-related protein 3R-1 (S. indicum) \\
\hline $\operatorname{RgMYB19}$ & Unigene19140 & 1451 & 419 & $100 \%, 3 \times 10^{-155}, 55 \%, \mathrm{XP} \_011076754.1$, uncharacterized protein $($ S. indicum $)$ \\
\hline $\operatorname{Rg} M Y B 20$ & Unigene9854 & 1257 & 303 & $100 \%, 2 \times 10^{-116}, 62 \%, \mathrm{XP}_{-} 011088207.1$, transcription factor MYB44-like $(S$. indicum $)$ \\
\hline $\operatorname{RgMYB21}$ & CL9364Contig2 & 1570 & 338 & $100 \%, 4 \times 10^{-162}, 74 \%, X_{-} 011074411.1$, transcription factor MYB44-like (S. indicum) \\
\hline $\operatorname{Rg} M Y B 22$ & CL6089Contig2 & 1303 & 307 & $86 \%, 1 \times 10^{-142}, 70 \%, \mathrm{XP}_{-} 011099313.1$, transcription factor MYB44-like $(S$. indicum $)$ \\
\hline $\operatorname{Rg} M Y B 23$ & Unigene11755 & 1536 & 374 & $84 \%, 6 \times 10^{-161}, 80 \%, \mathrm{XP}_{-} 011099313.1$, transcription factor MYB44-like (S. indicum) \\
\hline $\operatorname{RgMYB24}$ & CL173Contig2 & 1384 & 335 & $100 \%, 0.0,79 \%, \mathrm{XP} \_011077649.1$, transcription factor AS1 (S. indicum) \\
\hline $\operatorname{RgMYB25}$ & Unigene 5357 & 1478 & 387 & $96 \%, 0.0,84 \%$, XP_011097206.1, protein rough sheath 2 homolog $($ S. indicum $)$ \\
\hline
\end{tabular}


Table 1. Cont.

\begin{tabular}{|c|c|c|c|c|}
\hline Gene Name & Gene ID & $\begin{array}{l}\text { Gene } \\
\text { Length/bp }\end{array}$ & $\begin{array}{l}\text { Amino } \\
\text { Acid } \\
\text { Length }\end{array}$ & Blast Results (Query Cover, $e$-Value, Identities, Accession No., Description, (Species)) \\
\hline $\operatorname{RgMYB27}$ & CL5870Contig1 & 2525 & 697 & 99\%, 0.0, 74\%, XP_011085026.1, telomere repeat-binding protein 4 (S. indicum) \\
\hline $\operatorname{Rg} M Y B 28$ & CL7811Contig2 & 1869 & 593 & $100 \%, 0.0,73 \%$, XP_011094643.1, uncharacterized protein $(S$. indicum $)$ \\
\hline $\operatorname{Rg} M Y B 29$ & Unigene9541 & 836 & 233 & $100 \%, 3 \times 10^{-105}, 69 \%, \mathrm{XP} \_011094644.1$, uncharacterized protein $(S$. indicum $)$ \\
\hline $\operatorname{RgMYB30}$ & Unigene18531 & 2173 & 616 & $100 \%, 0.0,73 \%$, XP_011079786.1, uncharacterized protein $(S$. indicum $)$ \\
\hline $\operatorname{Rg} M Y B 31$ & CL4303Contig4 & 1484 & 302 & $100 \%, 8 \times 10^{-161}, 90 \%, \mathrm{XP} \_011088080.1$, telomere repeat-binding factor $1(S$. indicum $)$ \\
\hline $\operatorname{Rg} M Y B 32$ & Unigene21243 & 1440 & 300 & $100 \%, 2 \times 10^{-145}, 84 \%, X_{-} 011088080.1$, telomere repeat-binding factor 1 (S. indicum) \\
\hline $\operatorname{Rg} M Y B 33$ & CL4502Contig2 & 1359 & 295 & $100 \%, 2 \times 10^{-126}, 75 \%, X_{-} 011099389.1$, telomere repeat-binding factor 1 (S. indicum) \\
\hline $\operatorname{Rg} M Y B 34$ & CL4502Contig3 & 1226 & 294 & $100 \%, 8 \times 10^{-138}, 82 \%, \mathrm{XP}_{-} 011099389.1$ telomere repeat-binding factor 1 (S. indicum) \\
\hline $\operatorname{RgMYB35}$ & CL3772Contig3 & 1175 & 328 & $100 \%, 3 \times 10^{-176}, 73 \%, \mathrm{XP}_{0} 011095766.1$, MYB family transcription factor APL (S. indicum) \\
\hline $\operatorname{Rg} M Y B 36$ & Unigene13894 & 1053 & 272 & $68 \%, 3 \times 10^{-111}, 91 \%$, XP_011092595.1, MYB family transcription factor APL (S. indicum) \\
\hline $\operatorname{RgMYB37}$ & CL3105Contig2 & 714 & 187 & $100 \%, 5 \times 10^{-103}, 82 \%, \mathrm{XP} \_011097737.1$, transcription factor Divaricata-like $(S$. indicum $)$ \\
\hline $\operatorname{Rg} M Y B 38$ & CL725Contig2 & 1561 & 288 & $100 \%, 5 \times 10^{-177}, 88 \%$, XP_011094729.1, protein REVEILLE 8 (S. indicum) \\
\hline $\operatorname{RgMYB39}$ & CL5749Contig2 & 1086 & 266 & $100 \%, 6 \times 10^{-133}, 83 \%, \mathrm{XP} \_011071772.1$, transcription factor MYB1R1 (S. indicum) \\
\hline $\operatorname{RgMYB40}$ & CL613Contig1 & 1138 & 260 & $100 \%, 2 \times 10^{-111}, 72 \%, \mathrm{XP} 011071772.1$, transcription factor MYB1R1 (S. indicum) \\
\hline
\end{tabular}




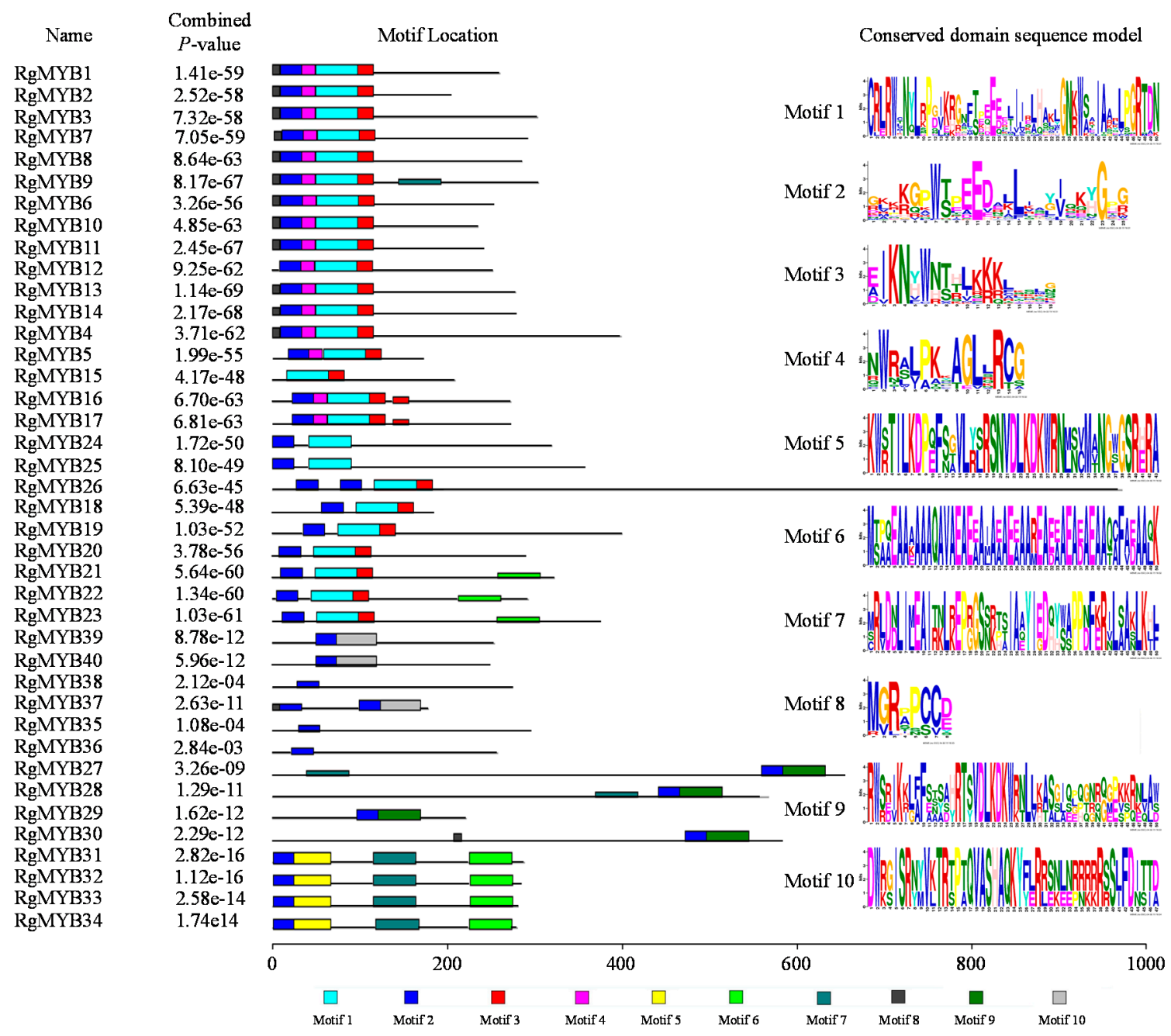

Figure 1. Schematic representation of conserved motifs in the MYB proteins of $R$. glutinosa, which were elucidated by MEME. Each motif is represented by a number in the colored box. The black lines represent the nonconserved sequences. The scale bar represents 200 amino acids.

Multiple amino acid sequence alignments using the BLASTp program and Clustal Omega showed that the $40 \mathrm{R}$. glutinosa MYBs were separated into three groups based on sequence similarity of the conserved MYB domain (Figure S1). Further sequence alignment revealed that the 26 proteins of group I shared the R2 and R3 MYB repeats and contained characteristic amino acids, including a series of evenly distributed and highly conserved Trp (W) residues (Figure 2A). As shown in Figure 2, these Trp (W) residues, except the first Trp (W) residues of the R3 repeat, are highly conserved in R. glutinosa MYB DBDs. In addition, RgMYB26 has another MYB repeat belonging to the R1R2R3 MYB protein. The six proteins of group II shared a MYB domain similar to the second MYB repeat of tomato LeMYBI [43], which acts as a transcriptional activator in yeast and plants and contains a SHAQKYF amino acid signature motif in the MYB-like repeat (Figure 2B). The sequence alignment of group III proteins indicated that eight MYBs contained a MYB-containing region of TRFL proteins that bound to the human telomeric DNA repeat sequence [44]. The most highly conserved region within all of the TRFL proteins, VDLKDKWR, lies within the telobox consensus sequence (Figure 2C). 


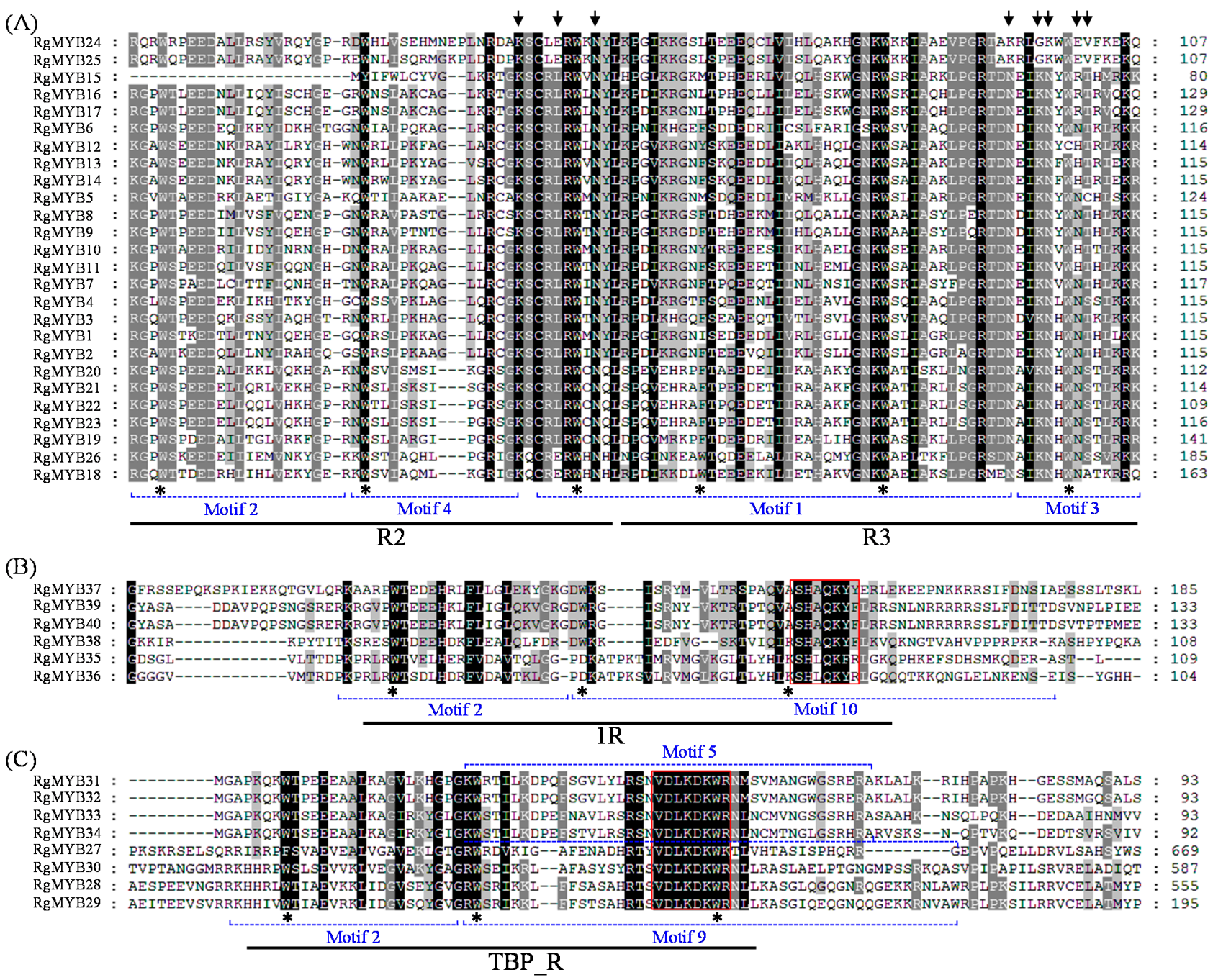

Figure 2. Highly conserved amino acid residues are present among the R2R3 (A), 1R (B) and TBP_R (C) domains of the $R$. glutinosa MYBs. The three regularly spaced Trp residues present in each repeat of the MYBs are labeled with asterisks. The amino acid sequences are aligned, and gaps (dots) have been introduced to maximize the alignment. The shading of the alignment presents identical residues in black, conserved residues in dark gray, and similar residues in light gray. The positions corresponding to base-contacting residues of the human MYBs are marked with arrows. The highly conserved SHAQKYF motifs in 1R and VDLKDKWR motif in TBP_R are boxed. Position of motif 2 plus motif 4 and part of motif 1 in Figure 1 relative to the R2 domain, motif 1 plus motif 3 relative to the $\mathrm{R} 3$ domain, motif 2 plus motif 10 relative to $1 \mathrm{R}$ domain, motif 2 plus motif 5 or motif 9 relative to TBP_R domain.

To learn more about the structures of the MYB genes, we performed protein homology modeling on three $R$. glutinosa MYB proteins using SWISS-MODEL. We used the structure of the A. thaliana MYB proteins as templates. A typical MYB domain is composed of a three- $\alpha$-helix bundle, which is thought to participate in binding DNA [45]. The generated molecular model showed that RgMYB40, RgMYB1, and RgMYB26 have three, six and nine $\alpha$-helices, respectively (Figure S2). 


\subsection{Phylogenetic Analysis of the MYB Proteins}

To further examine the evolutionary relationship among $R$. glutinosa, Arabidopsis and rice MYB proteins, the complete amino acid sequences of all MYB proteins were subjected to a multiple sequence alignment using the MAFFT program. The multiple sequence alignment file was then used to construct an unrooted phylogenetic tree using MEGA6.06 [46] by employing the neighbor-joining method. As shown in Figure 3, the phylogenetic tree divided the MYBs into four groups (groups I through IV). Group IV constitutes the largest clade with $58 \mathrm{MYBs,} \mathrm{followed} \mathrm{by} \mathrm{group} \mathrm{III} \mathrm{(17} \mathrm{proteins).}$ In addition, ten and five MYBs fall into groups I and II, respectively.

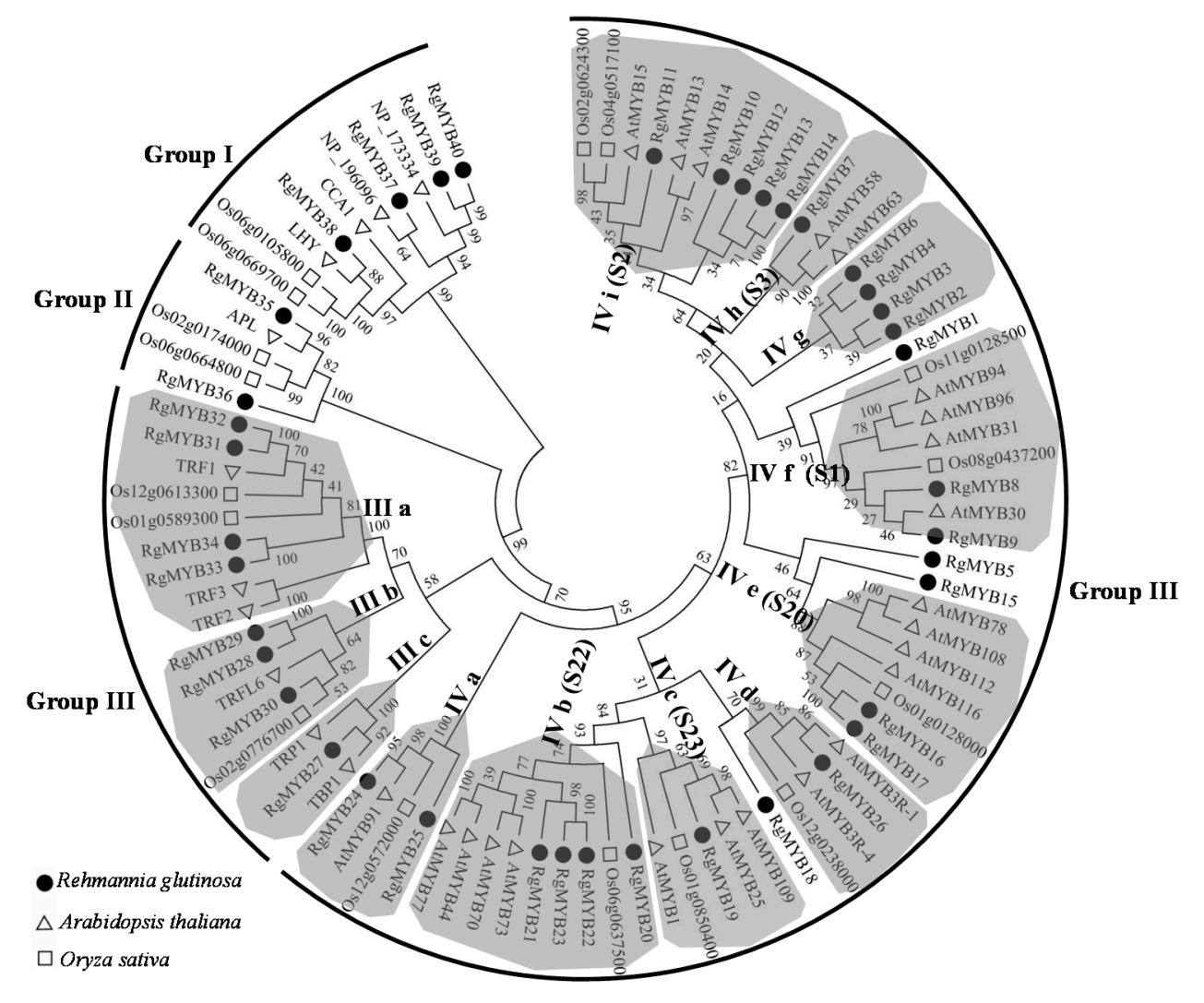

Figure 3. Phylogenetic tree of the MYB transcription factors from $R$. glutinosa, Arabidopsis and rice. The full-length sequences of the MYB proteins were aligned using MAFFT, and the phylogenetic tree was constructed using the neighbor-joining method in the MEGA6 software [46] (Tamura et al., 2013). Bootstrap values (shown at the corresponding nodes) were obtained from 1000 replicates and are reported as percentages. The colored shadow marks the subgroups of the MYBs.

As expected, most of the closely related members in the phylogenetic tree exhibited common motif compositions, suggesting that there are functional similarities among the MYB proteins within the same group. Specifically, Six RgMYBs (RgMYB37, 38, 39, 40, 35 and 36) were assembled together with LHY and APL $[47,48]$ in groups I and II, which represent the functional group of the regulation of circadian clock or phloem identity. Among them, RgMYB37 is a 2R MYB protein with two DBDs that are separated by long linker sequences. The other five RgMYB proteins are $1 \mathrm{R}$ MYB proteins with only one MYB domain with a SHAQKYF motif. The four RgMYB genes of subgroup IIIb and 
IIIc encode proteins with a single MYB domain at the C-terminus, similar to human TRF1 and Arabidopsis TBP1 [49,50]. The four RgMYB proteins of subgroup IIIa are members of the SMH (single MYB histone) protein family, which comprises double-stranded DNA-binding proteins that are specific to higher plants. They contain a MYB domain (motif 2 plus motif 5) at the N-terminus, a central H1/H5-like domain (motif 7) and a C-terminally located coiled-coil domain (motif 6). The SMH protein may specifically interact through the MYB domain with telomeric double-stranded DNA in vitro, while the central $\mathrm{H} 1 / \mathrm{H} 5$-like domain interacts non-specifically with DNA sequences and mediates protein-protein interactions $[51,52]$.

All the group IV proteins contain two repeats (R2R3), which are thought to have evolved from an $R 1 R 2 R 3-M Y B$ gene ancestor by the loss of the sequences encoding the $\mathrm{R} 1$ repeat and subsequent expansion of the gene family [8]. Group IV could be further divided into nine subgroup (named Iva-IVi). Similar to previous reports, the MYB genes with the same functions exhibited a tendency to cluster into one subgroup. For instance, subgroup IVb encompassed four $R$. glutinosa proteins homologous to AtMYB73/44 (Arabidopsis subgroup 22, S22), which regulates ABA-mediated stomatal closure in response to abiotic stresses [23]. Two proteins of subgroup IVa, RgMYB24 and RgMYB25, showed the highest sequence identity with AS1 and rough sheath 2 of $S$. indicum, respectively. AS1 and rough sheath 2 are orthologous genes from Arabidopsis and maize with similar molecular functions [53]. Two RgMYB proteins of subgroup IVe showed the highest identification with subgroup 20 of Arabidopsis, which is implicated in stress responses [22,24]. The five R. glutinosa proteins of subgroups IVi had high identity with subgroup 2 of Arabidopsis. The 3R-MYB protein, RgMYB26, was grouped into IVd with two Arabidopsis 3R-MYB proteins AtMYB3R-1 and AtMYB3R-4 [54], representing the functional group with proteins responsible for regulation of cytokinesis.

\subsection{Differential Expression of R. glutinosa MYB Genes in Various Tissues/Developmental Stages}

The gene expression pattern can provide important clues to gene function. We therefore analyzed the expression of $R$. glutinosa MYB genes in leaf and tuberous root by RNA-Seq. The expression profiles of all MYB family genes in R. glutinosa leaves and roots were evaluated using Pearson correlation Hierarchical Clustering with MultiExperiment Viewer (MeV) 4.9 software [55]. The results showed that 40 MYB genes were divided into two categories (Figure 4A and Table S2). Seventeen of the MYB genes were in group I and showed higher expression in leaves than in tuberous roots. The other 23 MYB genes formed group II and were highly expressed in tuberous roots. Among of them, eight MYB genes, including $\operatorname{RgMYB4}, \operatorname{Rg} M Y B 6, \operatorname{Rg} M Y B 7, \operatorname{RgMYB11}, \operatorname{Rg} M Y B 15, \operatorname{Rg} M Y B 22$, $\operatorname{RgMYB25}$ and $\operatorname{RgMYB33}$, were differently expressed genes.

To further study the potential roles of MYB genes in the formation of $R$. glutinosa tuberous roots, the expression profiles of 40 MYB genes in tuberous roots at the seedling stage (Z1), elongation and pre-expansion of tuberous root (Z2 and Z3) were evaluated using Euclidean distance Hierarchical Clustering with MeV 4.9 software. The results showed that 40 MYB genes fell into four groups (Figure 4B and Table S3). In particular, the expression levels of two MYB genes in group I, RgMYB23 and $\operatorname{Rg} M Y B 21$, were high in tuberous roots at all three stages. In contrast, the expression levels of three MYB genes, $\operatorname{Rg} M Y B 5, \operatorname{RgMYB} 40$ and $\operatorname{Rg} M Y B 39$, were very low during tuberous root development. Twenty-two MYB genes of group II showed high expression levels with minor differences, similar to 
constitutive expression. More than half of the 13 MYB genes in group III exhibited differential expression at different development stages of tuberous roots. In addition, K-means clustering was conducted to analyze the co-expression, and 40 MYB genes were divided into seven clusters based on their expression modulation (Figure S3). These clusters reflected the general trends and key transitional states during tuberous root development. Group I contains genes that first increased in expression at the Z2 stage and then decreased at the Z3 stage, while genes in group VII showed the opposite tendency. The highest expression levels of genes in group II and V were at the Z1 and Z3 stages, respectively. However, the transcriptional abundance of genes in groups III and VI were the lowest at the Z3 and Z1 stages, respectively.
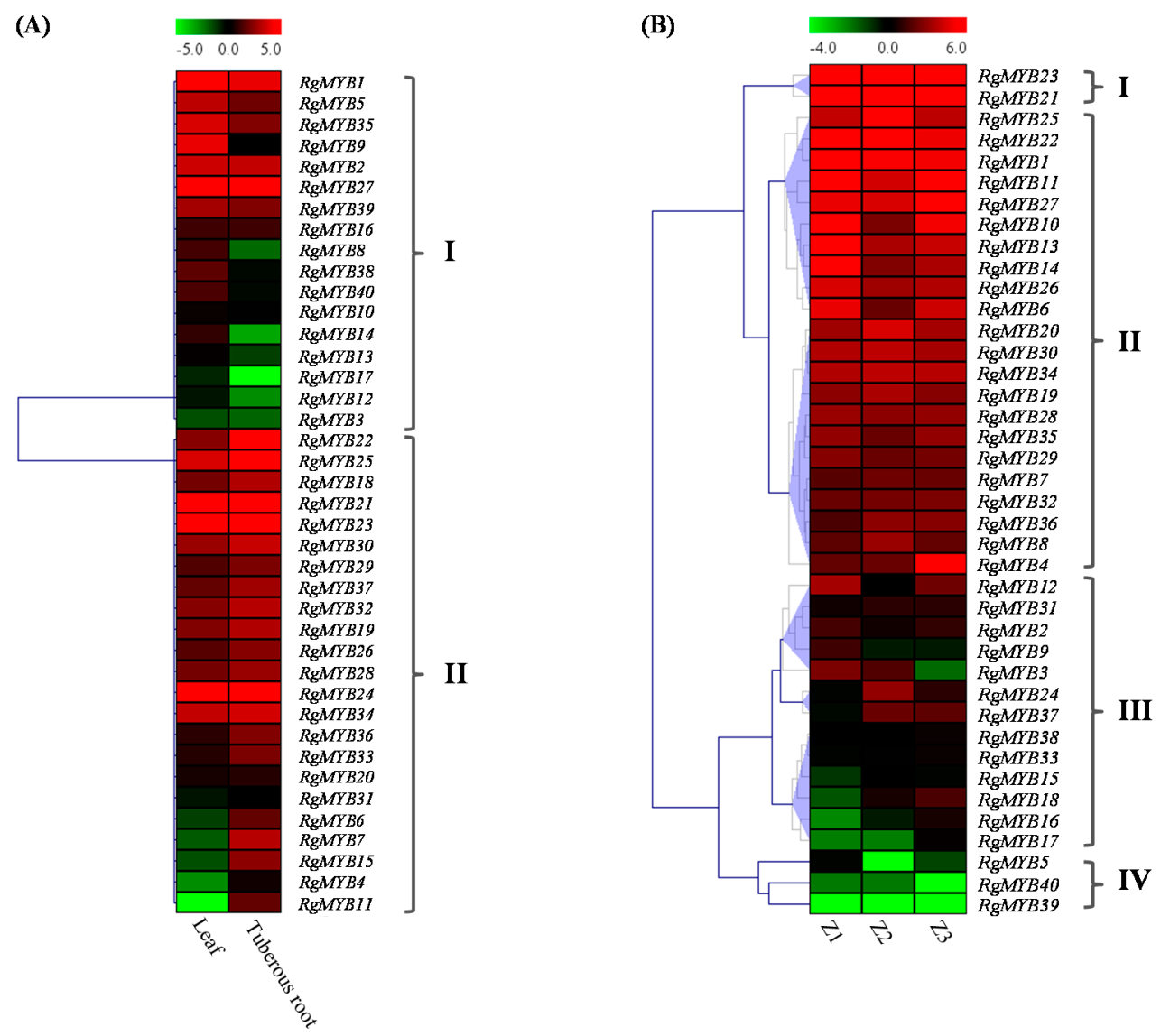

Figure 4. Expression profiles of $40 R$. glutinosa MYB family genes in different tissues/developments. (A) Expression levels of 40 MYBs in leaves and tuberous roots; (B) Expression levels of 40 MYB genes in tuberous roots at different stages. The color bar presented expression level of genes: green shows the low expression, and red color shows high expression level. Z1, Seedling stage; Z2, Elongation stage of tuberous root; and Z3, Pre-expanding stage of tuberous root. The heat maps were generated by MultiExperiment Viewer $(\mathrm{MeV})$.

\subsection{Expression Profiling of MYB Genes under Shading and Continuous Cropping Stresses}

R. glutinosa is a light-demanding plant species. Under shading conditions, development of R. glutinosa seedlings was inhibited, and the plants displayed decreased sizes of leaves and tuberous roots (Figure 5A). To identify MYB genes with a potential role in the shading response of plants, we 
analyzed the expression pattern of $R$. glutinosa MYB genes in response to shading. The expression of MYB genes was examined in the leaf and tuberous root under shading conditions by RNA-Seq. It was found that $70 \%$ (28) and $52.5 \%$ (21) of MYB genes were highly expressed to varying degrees in $90 \%$ shaded leaves and tuberous roots, respectively (Figure 5C and Table S4). Hierarchical clustering divided the 40 MYB genes into four groups based on their expression profiles in response to shading. Four and 11 MYB genes were differentially expressed in leaves and tuberous roots, respectively, after shading treatment. Among these genes, two MYB genes (RgMYB10 and RgMYB14) were strongly up regulated in both leaves and tuberous roots. Interestingly, under shading treatment, eight MYB genes (RgMYB1, RgMYB3, $\operatorname{RgMYB10,} \operatorname{Rg} M Y B 11, \operatorname{RgMYB13}, \operatorname{Rg} M Y B 14, \operatorname{Rg} M Y B 20$ and $\operatorname{Rg} M Y B 23$ ) were apparently induced, but only three genes $(\operatorname{Rg} M Y B 7, \operatorname{Rg} M Y B 15$ and $\operatorname{Rg} M Y B 22)$ were repressed. The qRT-PCR results agree with the results of the RNA-seq experiment (Figure S4).

(A)

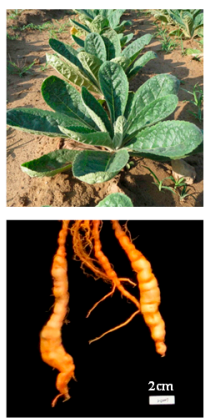

No shading

(B)

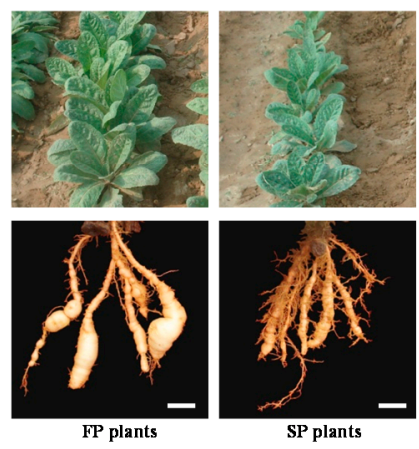

(C)

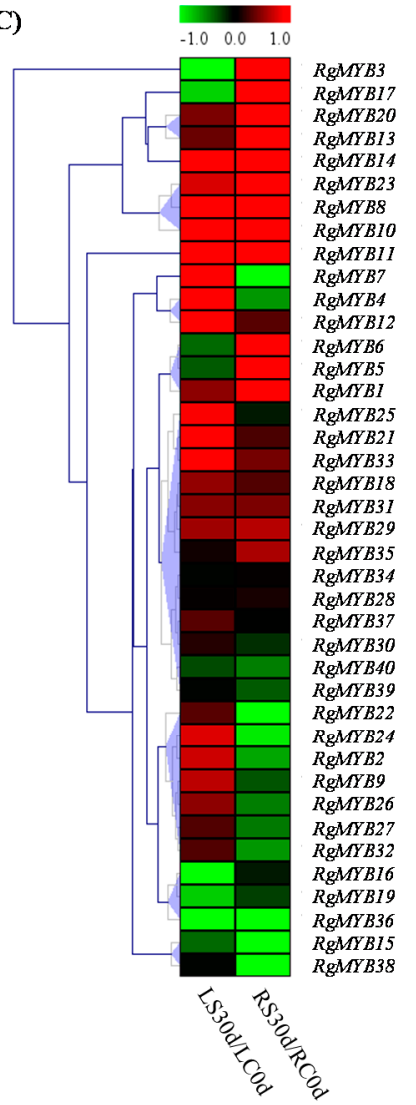

(D)
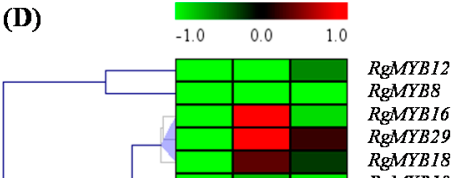

RgMYB8
RgMYBI6 RgMYB18 ${ }_{R \operatorname{Rg}}^{R g M P 13}$ ${ }_{R g M Y B 28}^{R}$ ${ }_{R g M Y B 30}^{R}$ RgMYB30
RgMYB26 RgMYB34 RgMYB32 RgMYMB27 RgMYBII RgMYB6 RgMYB3 RgMYBIO RgMYB335 RgMYB 36 RgMYB 9 RgMYBSI RgMYB2O RgMYB22 RgMYB37 RgMYBI RgMYB23 RgMVBII9 RgMYB 7 RgMYBA
RgMIYB33 RgMYMB 33
RgMYB 38 RgMYB 38
RgMYB2 RgMYBS
RgMYBI5 RgMYBDI5
RgMY2I RgMYB2I
RgMYB25 RgMYB25 RgMYM24
RgMYB5 RgMYB39 RgMYBI7
RgMYBA0 $\frac{2}{2} \frac{3}{5}$

Figure 5. Growth of $R$. glutinosa seeding and expression profiles of $R$. glutinosa MYB genes under abiotic stresses. (A) Effect of shading stress in $R$. glutinosa seedlings. Thirty-day-old seedlings were shaded and grown for an additional 35 days; (B) Phenotypes of 90-day-old $R$. glutinosa plants under continuous cropping conditions; White bars: $2 \mathrm{~cm}$; (C) Heat map showing the signal differences of 40 MYB genes in leaf and tuberous root under $90 \%$ shading compared to control; (D) Heat map showing the signal intensities of 40 MYB transcripts in tuberous roots at Z1, Z2 and Z3 stage under continuous cropping. The color bar presented expression level of genes: green shows the low expression, and red color shows high expression level. Z1, Seedling stage; Z2, Elongation stage of tuberous root; and Z3, Pre-expanding stage of tuberous root. The heat maps were generated by MultiExperiment Viewer (MeV). 
Continuously cropped $R$. glutinosa plants suffer from severe problems, including reduced biomass and decreased tuberous root products [35]. Continuous cropping significantly inhibited the growth of R. glutinosa, as indicated by reduced growth width, number of leaves per plant, leaf length, leaf width, tuberous root length, tuberous root width and tuberous fresh weight after two years of continuous cropping (Figure 5B). With the goal of identifying candidate continuous cropping responsive MYB genes, we analyzed the expression levels of 40 MYB genes from RNA-Seq data. There were $27(67.5 \%)$ MYB genes down-regulated in tuberous roots at the Z1 stage, and 12 (RgMYB6, $\operatorname{Rg} M Y B 8$, RgMYB11, RgMYB12, RgMYB13, RgMYB14, RgMYB26, RgMYB27, RgMYB28, RgMYB29, RgMYB30 and $\operatorname{RgMYB34}$ ) were differentially expressed genes (Figure 5D and Table S5). Curiously, unlike at the Z1 stage, up to $28(70 \%)$ MYB genes were up-regulated at the Z2 stage, but only one was differentially expressed. Interestingly, more than $80 \%$ of MYB genes were down-regulated at the Z3 stage. This result suggests that the response of MYB genes to the continuous cropping of $R$. glutinosa mainly occurred at the Z2 stage.

\subsection{Discussion}

In plants, MYB factors are one of the largest TF families [2,56]. More than 20 years ago, the first gene encoding a MYB TF in plants was identified. With the growing number of fully sequenced plant genomes, the identification of MYB genes has increased in recent times. Genome-wide analysis led to the identification of 155, 197 and 75 MYB genes in rice, Arabidopsis and sugar beet, respectively, which map to different chromosomes [57,58]. R2R3-MYB gene families have been annotated genome-wide in A. thaliana (126 members) [3], Z. mays (157 members) [59], O. sativa (102 members) [60], Vitis vinifera (117 members) [61], Populus trichocarpa (192 members) [62], G. max (244 members) [38] and Malus $\times$ domestica (222 members) [63]. However, no large dataset of MYB TFs is available for R. glutinosa.

In the present study, we identified 40 MYB genes with complete ORFs from $R$. glutinosa. Ten motifs were predicted by MEME, and seven of them were MYB/SANT domains according to SMART analysis. However, these motifs did not contain the basic features of whole MYB DBD domains. It may be that there were less well conserved DBD domain sequences among the diverse subgroup of R. glutinosa MYB proteins. Multiple amino acid sequence alignments using the BLASTp program and Clustal Omega showed that the 40 MYBs were separated into three groups based on sequence similarity of conserved MYB domain (Figure S5). Conserved domains of the three groups of MYB proteins were predicted by GLAM2 [64], and full-length MYB DBDs were identified that contained typical structure sequences. This result suggested that GLAM2 might even be used to analyze the domains of proteins in the same subfamily.

The six RgMYB proteins of groups I and II shared a MYB domain similar to the second MYB repeat of tomato LeMYBI [43], which acts as a transcriptional activator in yeast and plants. It contains a SHAQKYF amino acid signature motif in the MYB-like repeat (Figure 2B). A comparison of amino acid residues between the $1 \mathrm{R}$ regions of the six MYBs showed that the tryptophan residue is conserved at the first and second positions but not at the third position among most of the $1 \mathrm{R}$ regions. The sequence alignment of group III proteins indicated that the eight MYBs contained a MYB-containing region of TRFL proteins that bound to the human telomeric DNA repeat sequence [44]. The most 
highly conserved region within all of the TRFL proteins, VDLKDKWR [50], lies within the telobox consensus sequence (Figure 2C). We also observed that the third helix is better conserved than the first two helices in the DBD domain. It was previously demonstrated that the residues in the third helix are important for DNA binding by interacting with the DNA bases in the major groove when bound to DNA [65]. Further sequence alignment revealed that the $26 \mathrm{RgMYB}$ proteins of group IV shared the R2 and R3 MYB repeats and contained characteristic amino acids, including a series of evenly distributed and highly conserved $\operatorname{Trp}(\mathrm{W})$ residues (Figure 2A) that are known to play key roles in sequence-specific DNA binding $[38,66]$ and serve as landmarks of plant MYB proteins. As shown in Figure 2, these $\operatorname{Trp}(\mathrm{W})$ residues, except the first $\operatorname{Trp}(\mathrm{W})$ residue of the R3 repeat, are highly conserved in $R$. glutinosa MYB DBDs. In addition, RgMYB26 also has another MYB repeat, belonging to the R1R2R3 MYB protein.

To learn more about the structures of the MYB genes, we performed protein homology modeling on three MYB proteins of $R$. glutinosa using SWISS-MODEL. We used the solved structure of A. thaliana MYB proteins as templates. The typical MYB domain is comprised of a three- $\alpha$-helix bundle that is thought to bind DNA [45]. The generated molecular model showed that RgMYB40, RgMYB1, and RgMYB26 have three, six and nine $\alpha$-helices, respectively (Figure S2).

The topology of the MYB phylogeny indicates that some MYB genes in the same subgroup of Arabidopsis have the same function and that some MYB genes with similar functions are located in the same subgroup [67]. For instance, AtMYB11/PFG1, AtMYB12/PFG1 and AtMYB111/PFG3 (subgroup 7) control flavonol biosynthesis in all Arabidopsis tissues [14]. Similarly, AtMYB75, AtMYB90, AtMYB113 and AtMYB114 (subgroup 6) control anthocyanin biosynthesis in Arabidopsis vegetative tissues [15]. The R2R3-MYB proteins of Arabidopsis subgroup 12 regulate glucosinolate biosynthesis, and AtMYB28, AtMYB29 and AtMYB76 regulate the biosynthesis of aliphatic glucosinolates in aerial issues [10,15]. In this report, some $R$. glutinosa MYB proteins fell into a subgroup (Figure 3) that showed the highest homology to the Arabidopsis MYBs of another subgroup. Moreover, functions are broadly conserved for the MYB proteins of the same subgroup in different angiosperms. For example, two MYB orthologs, the snapdragon PHAN gene and the maize $r s 2$ gene, are located in subgroup G18. Both genes are involved in organ development $[68,69]$. R. glutinosa MYB proteins may share similar functions to Arabidopsis homologs in the same phylogenetic clades.

Functional studies on R2R3-MYB revealed that MYB genes in the same subgroup not only possess similar functions but are also co-expressed after stress treatments [70]. For example, AtMYB44, AtMYB77 and AtMYB73 of Arabidopsis subgroup 12 were up-regulated by wounding [71] and were also found to be up-regulated in $\operatorname{sos} 2$ mutants upon salt stress treatment [72]. The four MYB genes AtMYB44, AtMYB70, AtMYB73 and AtMYB77 showed similar temporal and spatial expression patterns and levels [70]. MYB genes in the same subgroup have redundant functions, as is supported by the co-expression phenomenon.

Most of the MYB genes in subgroup 2 are involved in Arabidopsis stress regulation [73]. In this study, five MYB genes (RgMYB10, RgMYB11, $\operatorname{RgMYB12,} \operatorname{Rg} M Y B 13$ and $\operatorname{Rg} M Y B 14)$ in subgroup IVi (Figure 3), which are homologous to Arabidopsis MYB genes in subgroup 2, showed similar expression patterns during the development of tuberous roots and in response to continuous cropping (Figures 4B and 5D). Most of them were differentially expressed under shading and continuous 
cropping conditions, implying that they could take part in the shading and continuous cropping responses in $R$. glutinosa.

Members of Arabidopsis subgroup 22 are involved not only in stresses response but also in plant development $[28,73]$. The expression levels of three orthologs of AtMYB73/44 (Arabidopsis subgroup 22), $\operatorname{RgMYB20}$, RgMYB21 and $\operatorname{RgMYB23}$, were up-regulated under shading conditions in leaf and/or tuberous root. However, only one gene ( $\operatorname{Rg} M Y B 22)$ was down regulated under shading. However, these genes were not differentially expressed under continuous cropping at the three $R$. glutinosa tuberous root stages. These genes may play important roles in the response to shading. AtMYB59 regulates root development through the control of cell cycle progression at the root tips [30]. Interestingly, we also found that an ortholog of AtMYB48/59 was up regulated in $R$. glutinosa tuberous root under shading and may also be involved in the shading response. Our results showed that $27(67.5 \%), 12(30 \%)$ and $33(82.5 \%)$ MYB genes were down-regulated in $R$. glutinosa tuberous roots at the Z1, Z2 and Z3 stages, respectively. Most $R$. glutinosa MYB genes were up-regulated in tuberous roots at the Z2 stage, suggesting that the Z2 stage might be the critical point in the formation of continuous cropping problems in R. glutinosa. Therefore, how MYB family genes participate in shading or continuous cropping stresses are intriguing issues for further study.

\section{Experimental Section}

\subsection{Plant Material and Sample Collection}

Our experimental R. glutinosa plants, "Wen 85-5", were grown in 2014 at Henan Agricultural University, Zhengzhou, China. The tuberous roots of $R$. glutinosa used for cultivation in all treatments were planted on 24 April 2014. Fields were maintained with locally normal production conditions. For shading experiments, more than 80 plants (four lines) were selected as untreated controls, while the other 80 plants were shaded to $10 \%$ of full sun with a high-density black-polypropylene shade cloth at 30 days after emergence. On 8 June (35 days after shading), leaves and tuberous roots of six randomly selected plants were collected from both control and treated seedlings. For the continuous cropping experiment, a group of seedlings was grown in a field in which $R$. glutinosa had not been planted for more than 10 years. The other group was grown in a field in which the same cultivar had been grown the previous year (planted on 25 April and harvested on 16 November, 2013). For convenience of description, we named the former group the first year (FP) planted $R$. glutinosa and the latter group as the second year (SP) replanted group. Each plant was regarded as a biological replicate. All samples mentioned above were immediately frozen in liquid nitrogen and stored at $-80{ }^{\circ} \mathrm{C}$ until use.

\subsection{Identification of Putative MYB $m R N A s$}

Sequences derived from the $R$. glutinosa expressed sequence tag (EST) database, a collection of more than 87,665 R. glutinosa leaf and root unigenes (mean length $554 \mathrm{bp}$ ), were assembled using SOAPdenovo software (http://soap.genomics.org.cn/) [74]. Coding sequence (CDS) of MYB genes from $A$. thaliana were used to search for $R$. glutinosa homologs in the EST database using basic local alignment (BLASTn). To remove redundancy, the sequences were assembled using the SeqMan function of the DNAStar software package and adjusted manually. Only the sequences that shared 
$>95 \%$ matches were considered redundant. The open reading frames (ORFs) were predicted using NCBI ORF Finder [75] and were translated into amino acid sequences. Finally, to confirm that the obtained sequences were MYB members, all of the non-redundant amino acid sequences of the primary identified MYB members were submitted to the website http://pfam.sanger.ac.uk to predict the MYB domains. Only the sequences that shared the MYB domain were confirmed to be MYB members. The $R$. glutinosa MYB sequences reported in this work have been submitted to GenBank and their accession numbers are KR780077-KR780116.

\subsection{Protein Structure and Phylogenetic Analysis}

To identify potential $R$. glutinosa MYB protein motifs, we used the MEME version 4.9.1 tool [76] with the following parameter settings: the distribution of motifs, 0 or 1 per sequence; maximum number of motifs to find, 10; minimum width of motif, 6; maximum width of motif, 50. In addition, only motifs with an $e$-value $\leq 1 \times 10^{-10}$ were retained for further analysis. Subsequently, the SMART (http://smart.embl.de/) [42] program was used to search for detected motifs in protein databases. The 3-D MYB protein models were constructed using the SWISS-MODEL programs [77].

The amino acid sequences of Arabidopsis (2 R1R2R3-MYB, 21 R2R3-MYB, and 11 MYB-related members) and rice (1 R1R2R3-MYB, 8 R2R3-MYB, and 7 MYB-related members) MYB proteins were downloaded from the NCBI database. The complete amino acid sequences of MYB proteins were used to construct phylogenetic trees. Phylogenetic trees were constructed with MEGA6.06 (www.megasoftware.net) by the neighbor-joining method and bootstrap analysis (1000 replicates) [46].

\subsection{Expression Profile Analysis of MYB Genes}

The total RNA from the leaf and the tuberous root was extracted using TRIzol reagent (Invitrogen, Carlsbad, CA, USA) and treated with DNase I to degrade any possible DNA contamination. Subsequently, the mRNA was enriched using oligo(dT) magnetic beads. The cDNA fragments were enriched by PCR amplification and then purified by magnetic beads. The library products were sent to BGI-Shenzhen for sequencing via the Ion Proton platform. Dirty raw reads were discarded via the following three steps: (1) reads with adaptors were removed; (2) reads with nucleotides less than 30 were removed; (3) remaining reads were trimmed (15 nt from 3' end); and (4) clean reads were obtained. The clean reads were mapped to the UniGene reference transcriptome using TRAP software [74]. Sequences with no more than 2 base pair mismatches were used in the alignment. The gene expression level was calculated using the RPKM (Reads Per Kilo bases per Million reads) method [78], and the formula is shown as follows:

$$
\mathrm{RPKM}=\frac{10^{6} \mathrm{C}}{\mathrm{NL} / 10^{3}}
$$

where $\mathrm{RPKM}(\mathrm{A})$ is the expression level of gene $\mathrm{A}, \mathrm{C}$ is the number of reads that uniquely aligned to gene $\mathrm{A}, \mathrm{N}$ is the total number of reads that uniquely aligned to all genes, and $\mathrm{L}$ is the number of bases of gene A. The $p$-value corresponds to a differential gene expression test. FDR (False Discovery Rate) is a method to determine the $p$-value threshold in multiple tests. We used "FDR $\leq 0.001$ and the absolute value of $\log _{2}$ Ratio $\geq 1$ " as the threshold to judge the significance of the gene expression 
differences. More stringent criteria with smaller FDRs and larger fold-change values can be used to identify differentially expressed genes.

\subsection{Quantitative Real-Time Polymerase Chain Reaction (PCR) Analysis}

Total RNA was extracted from different $R$. glutinosa fresh samples. Approximately $50 \mathrm{mg}$ tissue was collected and extracted using TRIzol reagent (Invitrogen). The RNA concentration was measured using a spectrophotometer, and integrity was ensured through analysis on a $1.5 \%(w / v)$ agarose gel. First-strand cDNA was synthesized in a $20 \mu \mathrm{L}$ mixture containing $1 \mu \mathrm{g}$ total RNA, $2 \mu \mathrm{L}$ of $50 \mu \mathrm{M}$ oligo-(dT)12-18 primers, $1 \mu \mathrm{L}$ RNase inhibitor and $1 \mu \mathrm{L}$ M-MLV reverse transcriptase (Invitrogen), incubated at $37^{\circ} \mathrm{C}$ for $50 \mathrm{~min}$, and then heated at $70{ }^{\circ} \mathrm{C}$ for $15 \mathrm{~min}$.

Real-time quantitative reverse transcription PCR (qRT-PCR) was performed for mediator subunit genes on an iCycler thermal cycler (iQ5, Bio-Rad, Hercules, CA, USA) Real Time PCR instrument. The RgTIP41 gene was used as endogenous control. The primers used are listed in Table S6. The PCR was conducted in a $25 \mu \mathrm{L}$ mixture containing $12.5 \mu \mathrm{L} \mathrm{SYBR}^{\circledR}$ Premix Ex Taq ${ }^{\mathrm{TM}}$ II (Tli RNaseH Plus) (Takara Bio, Dalian, China), $1 \mu \mathrm{L}$ cDNA template, and $1 \mu \mathrm{L}$ primers. The PCR program was: $95{ }^{\circ} \mathrm{C}$ for $30 \mathrm{~s}$, followed by 40 cycles of $95{ }^{\circ} \mathrm{C}$ for $5 \mathrm{~s}$ and $58{ }^{\circ} \mathrm{C}$ for $30 \mathrm{~s}$. Data were collected using the Bio-Rad iQ5 data detection system following the instructions. All reactions were performed in triplicate. After each assay, a dissociation kinetics analysis was performed to verify the specificity of the amplification products. Relative amounts of all mRNAs were calculated using the $2^{-\Delta \Delta C t}$ method [79]. The data for three biological replicates were analyzed using an analysis of variance (ANOVA) followed by Student's $t$-test $(p<0.05)$.

\section{Conclusions}

In the present study, 40 MYB genes were identified in $R$. glutinosa. These 40 genes were grouped into five categories on the basis of their phylogenetic relationships, which were well supported by additional conserved protein motifs. The expressions of some of these MYB genes varied under shading and continuous cropping stresses, suggesting that MYBs may play conserved and various roles, indicating some MYBs may be involved in R. glutinosa tuberous root development and abiotic stresses. These observations contribute valuable information for understanding the roles of MYB genes in regulating $R$. glutinosa tolerance to shading and continuous cropping.

\section{Supplementary Materials}

Supplementary materials can be found at http://www.mdpi.com/1422-0067/16/07/15009/s1.

\section{Acknowledgments}

This work was funded by the National Natural Science Foundation of China (Grant No. 81473299, 81274022), China Postdoctoral Science Foundation (Grant No. 2013M541977). 


\section{Author Contributions}

Fengqing Wang, Yanfei Suo and He Wei designed and performed the research, analyzed data and wrote the manuscript. Mingjie Li, Caixia Xie, Lina Wang and Xinjian Chen participated in analyzing the data and helped in drafting the manuscript. Zhongyi Zhang contributed to the interpretation of results and coordinated the study. All authors have read and approved the final draft.

\section{Conflicts of Interest}

The authors declare no conflict of interest.

\section{References}

1. Klempnauer, K.-H.; Gonda, T.J.; Bishop, J.M. Nucleotide sequence of the retroviral leukemia gene v-MYB and its cellular progenitor c-MYB: The architecture of a transduced oncogene. Cell 1982, 31, 453-463.

2. Dubos, C.; Stracke, R.; Grotewold, E.; Weisshaar, B.; Martin, C.; Lepiniec, L. MYB transcription factors in Arabidopsis. Trends Plant Sci. 2010, 15, 573-581.

3. Stracke, R.; Werber, M.; Weisshaar, B. The R2R3-MYB gene family in Arabidopsis thaliana. Curr. Opin. Plant Biol. 2001, 4, 447-456.

4. Rabinowicz, P.D.; Braun, E.L.; Wolfe, A.D.; Bowen, B.; Grotewold, E. Maize R2R3 Myb genes: Sequence analysis reveals amplification in the higher plants. Genetics 1999, 153, 427-444.

5. Paz-Ares, J.; Ghosal, D.; Wienand, U.; Peterson, P.A.; Saedler, H. The regulatory c1 locus of Zea mays encodes a protein with homology to myb proto-oncogene products and with structural similarities to transcriptional activators. EMBO J. 1987, 6, 3553-3558.

6. Jin, H.; Martin, C. Multifunctionality and diversity within the plant MYB-gene family. Plant Mol. Biol. 1999, 41, 577-585.

7. Rosinski, J.A.; Atchley, W.R. Molecular evolution of the MYB family of transcription factors: Evidence for polyphyletic origin. J. Mol. Evol. 1998, 46, 74-83.

8. Kranz, H.; Scholz, K.; Weisshaar, B. c-MYB oncogene-like genes encoding three MYB repeats occur in all major plant lineages. Plant J. 2000, 21, 231-235.

9. Penfield, S.; Meissner, R.C.; Shoue, D.A.; Carpita, N.C.; Bevan, M.W. MYB61 is required for mucilage deposition and extrusion in the Arabidopsis seed coat. Plant Cell 2001, 13, 2777-2791.

10. Gigolashvili, T.; Yatusevich, R.; Berger, B.; Muller, C.; Flugge, U.I. The R2R3-MYB transcription factor HAG1/MYB28 is a regulator of methionine-derived glucosinolate biosynthesis in Arabidopsis thaliana. Plant J. 2007, 51, 247-261.

11. Gigolashvili, T.; Engqvist, M.; Yatusevich, R.; Muller, C.; Flugge, U.I. HAG2/MYB76 and HAG3/MYB29 exert a specific and coordinated control on the regulation of aliphatic glucosinolate biosynthesis in Arabidopsis thaliana. New Phytol. 2008, 177, 627-642.

12. Gigolashvili, T.; Berger, B.; Flügge, U.I. Specific and coordinated control of indolic and aliphatic glucosinolate biosynthesis by R2R3-MYB transcription factors in Arabidopsis thaliana. Phytochem. Rev. 2009, 8, 3-13. 
13. Takos, A.M.; Jaffe, F.W.; Jacob, S.R.; Bogs, J.; Robinson, S.P.; Walker, A.R. Light-induced expression of a MYB gene regulates anthocyanin biosynthesis in red apples. Plant Physiol. 2006, 142, 1216-1232.

14. Stracke, R.; Ishihara, H.; Huep, G.; Barsch, A.; Mehrtens, F.; Niehaus, K.; Weisshaar, B. Differential regulation of closely related R2R3-MYB transcription factors controls flavonol accumulation in different parts of the Arabidopsis thaliana seedling. Plant J. 2007, 50, 660-677.

15. Gonzalez, A.; Zhao, M.; Leavitt, J.M.; Lloyd, A.M. Regulation of the anthocyanin biosynthetic pathway by the TTG1/bHLH/MYB transcriptional complex in Arabidopsis seedlings. Plant J. 2008, 53, 814-827.

16. Feng, F.; Li, M.; Ma, F.; Cheng, L. Phenylpropanoid metabolites and expression of key genes involved in anthocyanin biosynthesis in the shaded peel of apple fruit in response to sun exposure. Plant Physiol. Biochem. 2013, 69, 54-61.

17. Lai, L.B.; Nadeau, J.A.; Lucas, J.; Lee, E.K.; Nakagawa, T.; Zhao, L.; Geisler, M.; Sack, F.D. The Arabidopsis R2R3 MYB proteins FOUR LIPS and MYB88 restrict divisions late in the stomatal cell lineage. Plant Cell 2005, 17, 2754-2767.

18. Jakoby, M.J.; Falkenhan, D.; Mader, M.T.; Brininstool, G.; Wischnitzki, E.; Platz, N.; Hudson, A.; Hulskamp, M.; Larkin, J.; Schnittger, A. Transcriptional profiling of mature Arabidopsis trichomes reveals that NOECK encodes the MIXTA-like transcriptional regulator MYB106. Plant Physiol. 2008, 148, 1583-1602.

19. Kang, Y.H.; Kirik, V.; Hulskamp, M.; Nam, K.H.; Hagely, K.; Lee, M.M.; Schiefelbein, J. The MYB23 gene provides a positive feedback loop for cell fate specification in the Arabidopsis root epidermis. Plant Cell 2009, 21, 1080-1094.

20. Li, S.F.; Milliken, O.N.; Pham, H.; Seyit, R.; Napoli, R.; Preston, J.; Koltunow, A.M.; Parish, R.W. The Arabidopsis MYB5 transcription factor regulates mucilage synthesis, seed coat development, and trichome morphogenesis. Plant Cell 2009, 21, 72-89.

21. Xie, Z.; Lee, E.; Lucas, J.R.; Morohashi, K.; Li, D.; Murray, J.A.; Sack, F.D.; Grotewold, E. Regulation of cell proliferation in the stomatal lineage by the Arabidopsis MYB FOUR LIPS via direct targeting of core cell cycle genes. Plant Cell 2010, 22, 2306-2321.

22. Mengiste, T.; Chen, X.; Salmeron, J.; Dietrich, R. The BOTRYTIS SUSCEPTIBLE1 gene encodes an R2R3MYB transcription factor protein that is required for biotic and abiotic stress responses in Arabidopsis. Plant Cell 2003, 15, 2551-2565.

23. Jung, C.; Seo, J.S.; Han, S.W.; Koo, Y.J.; Kim, C.H.; Song, S.I.; Nahm, B.H.; Choi, Y.D.; Cheong, J.J. Over-expression of AtMYB44 enhances stomatal closure to confer abiotic stress tolerance in transgenic Arabidopsis. Plant Physiol. 2008, 146, 623-635.

24. Devaiah, B.N.; Madhuvanthi, R.; Karthikeyan, A.S.; Raghothama, K.G. Phosphate starvation responses and gibberellic acid biosynthesis are regulated by the MYB62 transcription factor in Arabidopsis. Mol. Plant 2009, 2, 43-58.

25. Seo, P.J.; Park, C.M. MYB96-mediated abscisic acid signals induce pathogen resistance response by promoting salicylic acid biosynthesis in Arabidopsis. New Phytol. 2010, 186, 471-483.

26. Millar, A.A.; Gubler, F. The Arabidopsis GAMYB-like genes, MYB33 and MYB65, are microRNA-regulated genes that redundantly facilitate anther development. Plant Cell 2005, 17, $705-721$. 
27. Zhang, Z.B.; Zhu, J.; Gao, J.F.; Wang, C.; Li, H.; Li, H.; Zhang, H.Q.; Zhang, S.; Wang, D.M.; Wang, Q.X.; et al. Transcription factor AtMYB103 is required for anther development by regulating tapetum development, callose dissolution and exine formation in Arabidopsis. Plant J. 2007, 52, 528-538.

28. Shin, R.; Burch, A.Y.; Huppert, K.A.; Tiwari, S.B.; Murphy, A.S.; Guilfoyle, T.J.; Schachtman, D.P. The Arabidopsis transcription factor MYB77 modulates auxin signal transduction. Plant Cell 2007, 19, 2440-2453.

29. Wang, X.; Niu, Q.W.; Teng, C.; Li, C.; Mu, J.; Chua, N.H.; Zuo, J. Over-expression of PGA37/MYB118 and MYB115 promotes vegetative-to-embryonic transition in Arabidopsis. Cell Res. 2009, 19, 224-235.

30. Mu, R.L.; Cao, Y.R.; Liu, Y.F.; Lei, G.; Zou, H.F.; Liao, Y.; Wang, H.W.; Zhang, W.K.; Ma, B.; $\mathrm{Du}, \mathrm{J} . Z$; et al. An R2R3-type transcription factor gene AtMYB59 regulates root growth and cell cycle progression in Arabidopsis. Cell Res. 2009, 19, 1291-1304.

31. Mandaokar, A. MYB108 acts together with MYB24 to regulate jasmonate-mediated stamen maturation in Arabidopsis. Plant Physiol. 2009, 149, 851-862.

32. Kwon, Y.; Kim, J.H.; Nguyen, H.N.; Jikumaru, Y.; Kamiya, Y.; Hong, S.W.; Lee, H. A novel Arabidopsis MYB-like transcription factor, $M Y B H$, regulates hypocotyl elongation by enhancing auxin accumulation. J. Exp. Bot. 2013, 64, 3911-3922.

33. Yang, Y.; Chen, X.; Chen, J.; Xu, H.; Li, J.; Zhang, Z. Differential miRNA expression in Rehmannia glutinosa plants subjected to continuous cropping. BMC Plant Biol. 2011, 11, 53.

34. Zhou, Y.; Zhang, Y.; Zhang, Y., Chen, Y; Bai, Y.; Wei, H.; Duan, H.; Zhou, C. Gene cloning, features of sequence, and analysis on temporal and spatial expression of Rehmannia glutinosa $\mathrm{f}$. hueichingensis 3-ketoacyl CoA-thiolase. Chin. Tradit. Herbal Drugs 2013, 44, 76-84.

35. Yang, Y.H.; Li, M.J.; Chen, X.J.; Wang, P.F.; Wang, F.Q.; Lin, W.X.; Yi, Y.J.; Zhang, Z.W.; Zhang, Z.Y. De novo characterization of the Rehmannia glutinosa leaf transcriptome and analysis of gene expression associated with replanting disease. Mol. Breed. 2014, 34, 905-915.

36. Wang, F.; Zhou, Y.; Huang, Y.; Li, M.; Tian, Y.; Feng, F.; Chen, X.; Zhang, Z. Cloning and expression analysis of the expansin gene RgEXPA10 in Rehmannia glutinosa. Acta Pharmacol. Sin. 2015, 50, 233-240.

37. Yang, A.; Dai, X.; Zhang, W.H. A R2R3-type MYB gene, OsMYB2, is involved in salt, cold, and dehydration tolerance in rice. J. Exp. Bot. 2012, 63, 2541-2556.

38. Du, H.; Yang, S.S.; Liang, Z.; Feng, B.R.; Liu, L.; Huang, Y.B.; Tang, Y.X. Genome-wide analysis of the MYB transcription factor superfamily in soybean. BMC Plant Biol. 2012, 12, 106.

39. Fornale, S.; Shi, X.; Chai, C.; Encina, A.; Irar, S.; Capellades, M.; Fuguet, E.; Torres, J.L.; Rovira, P.; Puigdomenech, P.; et al. ZmMYB31 directly represses maize lignin genes and redirects the phenylpropanoid metabolic flux. Plant J. 2010, 64, 633-644.

40. Altschul, S.F.; Madden, T.L.; Schaffer, A.A.; Zhang, J.; Zhang, Z.; Miller, W.; Lipman, D.J. Gapped BLAST and PSI-BLAST: A new generation of protein database search programs. Nucleic Acids Res. 1997, 25, 3389-3402.

41. Marchler-Bauer, A.; Lu, S.; Anderson, J.B.; Chitsaz, F.; Derbyshire, M.K.; de Weese-Scott, C.; Fong, J.H.; Geer, L.Y.; Geer, R.C.; Gonzales, N.R.; et al. CDD: A conserved domain database for the functional annotation of proteins. Nucleic Acids Res. 2011, 39, D225-D229. 
42. Letunic, I.; Doerks, T.; Bork, P. SMART: Recent updates, new developments and status in 2015. Nucleic Acids Res. 2015, 43, D257-D260.

43. Rose, A.; Meier, I.; Wienand, U. The tomato I-box binding factor LeMYBI is a member of a novel class of MYB-like proteins. Plant J. 1999, 20, 641-652.

44. Nishikawa, T.; Okamura, H.; Nagadoi, A.; Konig, P.; Rhodes, D.; Nishimura, Y. Solution structure of a telomeric DNA complex of human TRF1. Structure 2001, 9, 1237-1251.

45. Zhang, F.; Liu, X.; Zuo, K.; Sun, X.; Tang, K. Molecular cloning and expression analysis of a novel SANT/MYB gene from Gossypium barbadense. Mol. Biol. Rep. 2011, 38, 2329-2336.

46. Tamura, K.; Stecher, G.; Peterson, D.; Filipski, A.; Kumar, S. MEGA6: Molecular evolutionary genetics analysis version 6.0. Mol. Biol. Evol. 2013, 30, 2725-2729.

47. Alabadí, D.; Oyama, T.; Yanovsky, M.J.; Harmon, F.G.; Mas, P.; Kay, S.A. Reciprocal regulation between TOC1 and LHY/CCA1 within the Arabidopsis circadian clock. Science 2001, 293, $880-883$.

48. Bonke, M.; Thitamadee, S.; Mähönen, A.P.; Hauser, M.-T.; Helariutta, Y. APL regulates vascular tissue identity in Arabidopsis. Nature 2003, 426, 181-186.

49. Smogorzewska, A.; van Steensel, B.; Bianchi, A.; Oelmann, S.; Schaefer, M.R.; Schnapp, G.; de Lange, T. Control of human telomere length by TRF1 and TRF2. Mol. Cell. Biol. 2000, 20, 1659-1668.

50. Karamysheva, Z.N.; Surovtseva, Y.V.; Vespa, L.; Shakirov, E.V.; Shippen, D.E. A C-terminal MYB extension domain defines a novel family of double-strand telomeric DNA-binding proteins in Arabidopsis. J. Biol. Chem. 2004, 279, 47799-47807.

51. Dvorackova, M.; Rossignol, P.; Shaw, P.J.; Koroleva, O.A.; Doonan, J.H.; Fajkus, J. AtTRB1, a telomeric DNA-binding protein from Arabidopsis, is concentrated in the nucleolus and shows highly dynamic association with chromatin. Plant J. 2010, 61, 637-649.

52. Yun, J.H.; Lee, W.K.; Kim, H.; Kim, E.; Cheong, C.; Cho, M.H.; Lee, W. Solution structure of telomere binding domain of AtTRB2 derived from Arabidopsis thaliana. Biochem. Biophys. Res. Commun. 2014, 452, 436-442.

53. Illing, N.; Klak, C.; Johnson, C.; Brito, D.; Negrao, N.; Baine, F.; van Kets, V.; Ramchurn, K.R.; Seoighe, C.; Roden, L. Duplication of the Asymmetric Leaves1/Rough Sheath 2/Phantastica (ARP) gene precedes the explosive radiation of the Ruschioideae. Dev. Genes Evol. 2009, 219, 331-338.

54. Haga, N.; Kato, K.; Murase, M.; Araki, S.; Kubo, M.; Demura, T.; Suzuki, K.; Müller, I.; Voß, U.; Jürgens, G. R1R2R3-Myb proteins positively regulate cytokinesis through activation of KNOLLE transcription in Arabidopsis thaliana. Development 2007, 134, 1101-1110.

55. Saeed, A.I.; Bhagabati, N.K.; Braisted, J.C.; Liang, W.; Sharov, V.; Howe, E.A.; Li, J.; Thiagarajan, M.; White, J.A.; Quackenbush, J. TM4 microarray software suite. Methods Enzymol. 2006, 411, 134-193.

56. Romero, I.; Fuertes, A.; Benito, M.J.; Malpica, J.M.; Leyva, A.; Paz-Ares, J. More than 80 R2R3-MYB regulatory genes in the genome of Arabidopsis thaliana. Plant J. 1998, 14, 273-284.

57. Katiyar, A.; Smita, S.; Lenka, S.K.; Rajwanshi, R.; Chinnusamy, V.; Bansal, K.C. Genome-wide classification and expression analysis of MYB transcription factor families in rice and Arabidopsis. BMC Genomics 2012, 13, 544. 
58. Stracke, R.; Holtgrawe, D.; Schneider, J.; Pucker, B.; Sorensen, T.R.; Weisshaar, B. Genome-wide identification and characterisation of R2R3-MYB genes in sugar beet (Beta vulgaris). BMC Plant Biol. 2014, 14, 249.

59. Du, H.; Feng, B.R.; Yang, S.S.; Huang, Y.B.; Tang, Y.X. The R2R3-MYB transcription factor gene family in maize. PLOS ONE 2012, 7, e37463.

60. Yanhui, C.; Xiaoyuan, Y.; Kun, H.; Meihua, L.; Jigang, L.; Zhaofeng, G.; Zhiqiang, L.; Yunfei, Z.; Xiaoxiao, W.; Xiaoming, Q.; et al. The MYB transcription factor superfamily of Arabidopsis: Expression analysis and phylogenetic comparison with the rice MYB family. Plant Mol. Biol. 2006, 60, 107-124.

61. Matus, J.T.; Aquea, F.; Arce-Johnson, P. Analysis of the grape MYB R2R3 subfamily reveals expanded wine quality-related clades and conserved gene structure organization across Vitis and Arabidopsis genomes. BMC Plant Biol. 2008, 8, 83.

62. Wilkins, O.; Nahal, H.; Foong, J.; Provart, N.J.; Campbell, M.M. Expansion and diversification of the Populus R2R3-MYB family of transcription factors. Plant Physiol. 2009, 149, 981-993.

63. Cao, Z.H.; Zhang, S.Z.; Wang, R.K.; Zhang, R.F.; Hao, Y.J. Genome wide analysis of the apple MYB transcription factor family allows the identification of MdoMYB121 gene confering abiotic stress tolerance in plants. PLoS ONE 2013, 8, e69955.

64. Frith, M.C.; Saunders, N.F.; Kobe, B.; Bailey, T.L. Discovering sequence motifs with arbitrary insertions and deletions. PLoS Comput. Biol. 2008, 4, e1000071.

65. Ogata, K.; Morikawa, S.; Nakamura, H.; Sekikawa, A.; Inoue, T.; Kanai, H.; Sarai, A.; Ishii, S.; Nishimura, Y. Solution structure of a specific DNA complex of the MYB DNA-binding domain with cooperative recognition helices. Cell 1994, 79, 639-648.

66. Ogata, K.; Hojo, H.; Aimoto, S.; Nakai, T.; Nakamura, H.; Sarai, A.; Ishii, S.; Nishimura, Y. Solution structure of a DNA-binding unit of MYB: A helix-turn-helix-related motif with conserved tryptophans forming a hydrophobic core. Proc. Natl. Acad. Sci. USA 1992, 89, 6428-6432.

67. Jiang, C.; Gu, X.; Peterson, T. Identification of conserved gene structures and carboxy-terminal motifs in the MYB gene family of Arabidopsis and Oryza sativa L. ssp. indica. Genome Biol. 2004, 5, R46.

68. Waites, R.; Selvadurai, H.R.; Oliver, I.R.; Hudson, A. The PHANTASTICA gene encodes a MYB transcription factor involved in growth and dorsoventrality of lateral organs in Antirrhinum. Cell 1998, 93, 779-789.

69. Tsiantis, M.; Schneeberger, R.; Golz, J.F.; Freeling, M.; Langdale, J.A. The maize rough sheath2 gene and leaf development programs in monocot and dicot plants. Science 1999, 284, 154-156.

70. Fan, J.; Jiang, C.; Xing, J.; Dong, J. Structure and function of the 22nd subfamily in Arabidopsis R2R3-MYB family. Yi Chuan 2014, 36, 985-994.

71. Cheong, Y.H.; Chang, H.S.; Gupta, R.; Wang, X.; Zhu, T.; Luan, S. Transcriptional profiling reveals novel interactions between wounding, pathogen, abiotic stress, and hormonal responses in Arabidopsis. Plant Physiol. 2002, 129, 661-677.

72. Kamei, A.; Seki, M, Umezawa, T.; Ishida, J.; Satou, M.; Akiyama, K.; Zhu, J.; Shinozaki, K. Analysis of gene expression profiles in Arabidopsis salt overly sensitive mutants sos 2-1 and sos3-1. Plant Cell Environ. 2005, 28, 1267-1275. 
73. Agarwal, M.; Hao, Y.; Kapoor, A.; Dong, C.H.; Fujii, H.; Zheng, X.; Zhu, J.K. A R2R3 type MYB transcription factor is involved in the cold regulation of CBF genes and in acquired freezing tolerance. J. Biol. Chem. 2006, 281, 37636-37645.

74. Li, R.; Yu, C.; Li, Y.; Lam, T.W.; Yiu, S.M.; Kristiansen, K.; Wang, J. SOAP2: An improved ultrafast tool for short read alignment. Bioinformatics 2009, 25, 1966-1967.

75. Wheeler, D.L.; Chappey, C.; Lash, A.E.; Leipe, D.D.; Madden, T.L.; Schuler, G.D.; Tatusova, T.A.; Rapp, B.A. Database resources of the national center for biotechnology information. Nucleic Acids Res. 2000, 28, 10-14.

76. Bailey, T.L.; Boden, M.; Buske, F.A.; Frith, M.; Grant, C.E.; Clementi, L.; Ren, J.; Li, W.W.; Noble, W.S. MEME SUITE: Tools for motif discovery and searching. Nucleic Acids Res. 2009, 37, W202-W208.

77. Biasini, M.; Bienert, S.; Waterhouse, A.; Arnold, K.; Studer, G.; Schmidt, T.; Kiefer, F.; Cassarino, T.G.; Bertoni, M.; Bordoli, L.; et al. SWISS-MODEL: Modelling protein tertiary and quaternary structure using evolutionary information. Nucleic Acids Res. 2014, 42, W252-W258.

78. Mortazavi, A.; Williams, B.A.; McCue, K.; Schaeffer, L.; Wold, B. Mapping and quantifying mammalian transcriptomes by RNA-Seq. Nat. Methods 2008, 5, 621-628.

79. Schmittgen, T.D.; Livak, K.J. Analyzing real-time PCR data by the comparative $C_{\mathrm{t}}$ method. Nat. Protoc. 2008, 3, 1101-1108.

(C) 2015 by the authors; licensee MDPI, Basel, Switzerland. This article is an open access article distributed under the terms and conditions of the Creative Commons Attribution license (http://creativecommons.org/licenses/by/4.0/). 